\title{
Crystal structure of bis(1-phenyl-5-cyclohexyl-pentane-1,3-dionato) copper(II), $\left[\mathrm{Cu}\left(\mathrm{C}_{17} \mathrm{H}_{21} \mathrm{O}_{2}\right)_{2}\right]$
}

\author{
U. Schilde, W. Mickler and E. Uhlemann \\ Universität Potsdam. Institut für Anorganische Chemie und Didaktik der Chemie. Postfach 6015 53, Am Neuen Palais 10. D-14415 Potsdam, Germany
}

Received June 21, 1996. CSD-No. 402542

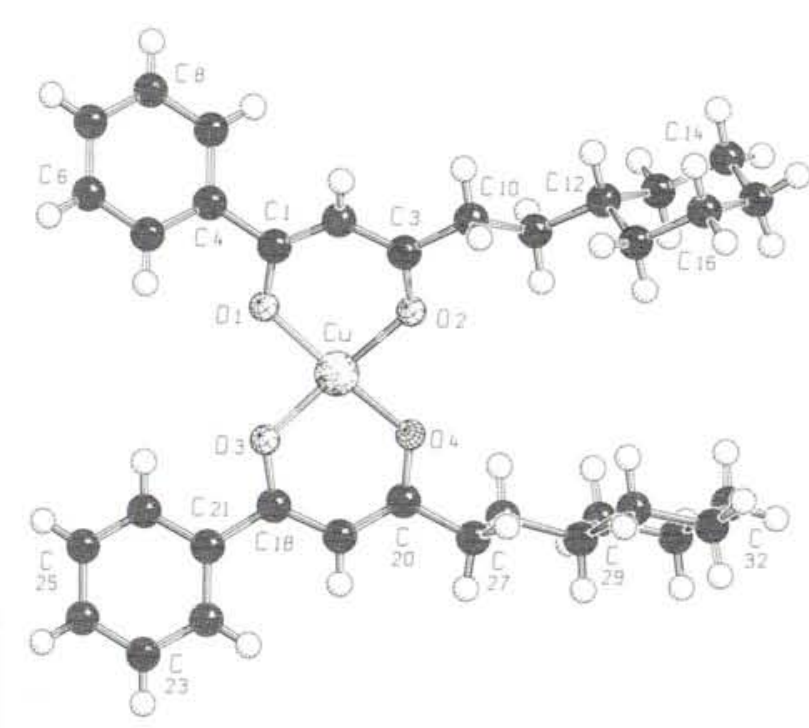

Source of material: The complex was prepared by the reaction of copper acetate with 1-phenyl- 5-cyclohexyl-pentane-1,3-dione in ethanol (see ref. 2). The compounds were purified in chloroform and the crystals can be obtained from hexane.

The unexpected cis-configuration of a complex isolated from an extraction process was recently reported (see ref. 1). For the further study of this effect crystals of a long-chain $\beta$-diketone copper complex were now prepared by reaction in homogeneous solution. The ligands are in a square planar arrangement around $\mathrm{Cu}$ and a cis-configuration was confirmed as well.

Table 1. Parameters used for the X-ray data collection

$\begin{array}{ll}\text { Crystal: } & \text { dark-green prism, size } 0.23 \times 0.53 \times 0.65 \mathrm{~mm} \\ \text { Wavelength: } & \text { Mo } K_{\alpha} \text { radiation }(0.71073 \AA) \\ \mu: & 7.51 \mathrm{~cm}^{-1} \\ \text { Diffractometer: } & \text { Stoe STADI-4 } \\ \text { Scan mode: } & \omega / 2 \theta \\ \mathrm{T}_{\text {measurement: }} & 298 \mathrm{~K} \\ 2 \theta_{\text {max: }} & 64^{\circ} \\ \mathrm{N}(\text { hkl })_{\text {unique: }} & 3540 \\ \text { Criterion for } I_{\mathrm{o}}: & I_{0}>2 \sigma\left(I_{0}\right) \\ \mathrm{N}(\text { param }) \text { refined: } & 360 \\ \text { Program: } & \text { SHELXL-93 }\end{array}$

$\mathrm{C}_{34} \mathrm{H}_{42} \mathrm{CuO}_{4}$, monoclinic, $P_{2} / / c$ (No. 14), $a=17.698(5) \AA$, $b=9.778(5) \AA, c=19.37(1) \AA, \beta=114.55(2)^{\circ}, V=3049.0 \AA^{3}$, $Z=4, R(F)=0.042, R_{w}\left(F^{2}\right)=0.117$.

Table 2. Final atomic coordinates and displacement parameters (in $\AA^{2}$ )

\begin{tabular}{|c|c|c|c|c|c|}
\hline Atom & Site & $x$ & $y$ & $z$ & $U_{\text {iso }}$ \\
\hline $\mathbf{H}(2 \mathrm{~A})$ & $4 e$ & $0.447(2)$ & $0.241(4)$ & $0.541(2)$ & $0.04(1)$ \\
\hline$H(5 A)$ & $4 e$ & $0.6961(3)$ & $0.3408(5)$ & $0.6019(3)$ & 0.073 \\
\hline$H(6 A)$ & $4 e$ & $0.7943(3)$ & $0.2242(6)$ & $0.7034(3)$ & 0.092 \\
\hline$H(7 A)$ & $4 e$ & $0.7636(3)$ & $0.0136(6)$ & $0.7377(3)$ & 0.089 \\
\hline $\mathrm{H}(8 \mathrm{~A})$ & $4 e$ & $0.6341(3)$ & $-0.0765(6)$ & $0.6745(3)$ & 0.093 \\
\hline$H(9 A)$ & $4 e$ & $0.5326(3)$ & $0.0439(5)$ & $0.5786(3)$ & 0.076 \\
\hline $\mathrm{H}(10 \mathrm{~A})$ & $4 e$ & $0.3035(3)$ & $0.2559(6)$ & $0.4758(3)$ & 0.088 \\
\hline$H(10 B)$ & $4 e$ & $0.2997(3)$ & $0.4157(6)$ & $0.4733(3)$ & 0.088 \\
\hline H(11A) & $4 e$ & $0.2429(3)$ & $0.2475(6)$ & $0.3467(3)$ & 0.098 \\
\hline $\mathrm{H}(11 \mathrm{~B})$ & $4 e$ & $0.2414(3)$ & $0.4072(6)$ & $0.3428(3)$ & 0.098 \\
\hline$H(12 A)$ & $4 e$ & $0.1517(3)$ & $0.2701(5)$ & $0.4123(3)$ & 0.079 \\
\hline $\mathbf{H}(13 \mathrm{~A})$ & $4 e$ & $0.1034(3)$ & $0.1818(7)$ & $0.2913(4)$ & 0.118 \\
\hline$H(13 B)$ & $4 e$ & $0.0862(3)$ & $0.3294(7)$ & $0.2567(4)$ & 0.118 \\
\hline$H(14 A)$ & $4 e$ & $-0.0390(3)$ & $0.2366(7)$ & $0.2467(4)$ & 0.125 \\
\hline H(14B) & $4 e$ & $-0.0011(3)$ & $0.2055(7)$ & $0.3341(4)$ & 0.125 \\
\hline $\mathrm{H}(15 \mathrm{~A})$ & $4 e$ & $-0.0793(3)$ & $0.3960(8)$ & $0.3122(4)$ & 0.134 \\
\hline H(15B) & $4 e$ & $-0.0321(3)$ & $0.4632(8)$ & $0.2680(4)$ & 0.134 \\
\hline$H(16 A)$ & $4 e$ & $0.0354(3)$ & $0.4146(8)$ & $0.4250(4)$ & 0.152 \\
\hline $\mathrm{H}(16 \mathrm{~B})$ & $4 e$ & $0.0175(3)$ & $0.5591(8)$ & $0.3862(4)$ & 0.152 \\
\hline$H(17 A)$ & $4 e$ & $0.1224(3)$ & $0.5316(6)$ & $0.3457(4)$ & 0.112 \\
\hline $\mathrm{H}(17 \mathrm{~B})$ & $4 e$ & $0.1603(3)$ & $0.5032(6)$ & $0.4333(4)$ & 0.112 \\
\hline $\mathrm{H}(19 \mathrm{~A})$ & $4 e$ & $0.545(3)$ & $0.737(5)$ & $0.250(3)$ & $0.08(2)$ \\
\hline$H(22 A)$ & $4 e$ & $0.6644(3)$ & $0.8147(4)$ & $0.2796(3)$ & 0.067 \\
\hline$H(23 A)$ & $4 e$ & $0.8001(3)$ & $0.8628(5)$ & $0.3030(3)$ & 0.080 \\
\hline$H(24 A)$ & $4 e$ & $0.9061(3)$ & $0.7264(6)$ & $0.3811(3)$ & 0.091 \\
\hline$H(25 A)$ & $4 e$ & $0.8767(3)$ & $0.5351(6)$ & $0.4354(3)$ & 0.086 \\
\hline $\mathrm{H}(26 \mathrm{~A})$ & $4 e$ & $0.7428(3)$ & $0.4882(5)$ & $0.4159(2)$ & 0.072 \\
\hline $\mathrm{H}(27 \mathrm{~A})$ & $4 e$ & $0.3389(3)$ & $0.7304(6)$ & $0.2105(3)$ & 0.098 \\
\hline $\mathrm{H}(27 \mathrm{~B})$ & $4 e$ & $0.4014(3)$ & $0.7815(6)$ & $0.1776(3)$ & 0.098 \\
\hline$H(28 A)$ & $4 e$ & $0.3364(3)$ & $0.5182(6)$ & $0.1496(3)$ & 0.105 \\
\hline $\mathrm{H}(28 \mathrm{~B})$ & $4 e$ & $0.3838(3)$ & $0.5918(6)$ & $0.1077(3)$ & 0.105 \\
\hline $\mathrm{H}(29 \mathrm{~A})$ & $4 e$ & $0.2690(3)$ & $0.7370(5)$ & $0.0458(3)$ & 0.092 \\
\hline$H(30 A)$ & $4 e$ & $0.2323(4)$ & $0.4575(6)$ & $0.0226(3)$ & 0.110 \\
\hline $\mathrm{H}(30 \mathrm{~B})$ & $4 e$ & $0.2782(4)$ & $0.5440(6)$ & $-0.0164(3)$ & 0.110 \\
\hline$H(31 A)$ & $4 e$ & $0.1583(4)$ & $0.6650(7)$ & $-0.0853(3)$ & 0.121 \\
\hline $\mathrm{H}(31 \mathrm{~B})$ & $4 e$ & $0.1399(4)$ & $0.5082(7)$ & $-0.0999(3)$ & 0.121 \\
\hline$H(32 A)$ & $4 e$ & $0.0750(4)$ & $0.5048(8)$ & $-0.0177(4)$ & 0.138 \\
\hline $\mathrm{H}(32 \mathrm{~B})$ & $4 e$ & $0.0330(4)$ & $0.6176(8)$ & $-0.0797(4)$ & 0.138 \\
\hline$H(33 A)$ & $4 e$ & $0.0634(4)$ & $0.7021(8)$ & $0.0441(4)$ & 0.133 \\
\hline H(33B) & $4 e$ & $0.1077(4)$ & $0.7891(8)$ & $0.0036(4)$ & 0.133 \\
\hline$H(34 A)$ & $4 e$ & $0.2055(4)$ & $0.7427(6)$ & $0.1268(3)$ & 0.113 \\
\hline H(34B) & $4 e$ & $0.1860(4)$ & $0.5860(6)$ & $0.1161(3)$ & 0.113 \\
\hline
\end{tabular}


Table 3. Final atomic coordinates and displacement parameters (in $\AA^{2}$ )

\begin{tabular}{|c|c|c|c|c|c|c|c|c|c|c|}
\hline Atom & Site & $x$ & $y$ & $z$ & $U_{11}$ & $U_{23}$ & $U_{33}$ & $U_{12}$ & $U_{13}$ & $U_{23}$ \\
\hline $\mathrm{Cu}$ & $4 e$ & $0.49865(3)$ & $0.45798(5)$ & $0.38747(3)$ & $0.0476(4)$ & $0.0433(4)$ & $0.0454(3)$ & $0.0012(2)$ & $0.0199(2)$ & $0.0084(2)$ \\
\hline$C(1)$ & $4 e$ & $0.5397(3)$ & $0.2827(4)$ & $0.5156(2)$ & $0.048(3)$ & $0.038(3)$ & $0.046(3)$ & $0.004(2)$ & $0.023(2)$ & $0.001(2)$ \\
\hline $\mathrm{C}(2)$ & $4 e$ & $0.4589(3)$ & $0.2799(5)$ & $0.5065(3)$ & $0.051(4)$ & $0.062(3)$ & $0.050(3)$ & $0.005(2)$ & $0.026(2)$ & $0.018(2)$ \\
\hline$C(3)$ & $4 e$ & $0.3917(3)$ & $0.3401(4)$ & $0.4477(2)$ & $0.045(3)$ & $0.057(3)$ & $0.055(3)$ & $-0.001(2)$ & $0.023(2)$ & $0.008(2)$ \\
\hline$C(4)$ & $4 e$ & $0.6038(3)$ & $0.2050(4)$ & $0.5791(2)$ & $0.052(3)$ & $0.044(3)$ & $0.045(2)$ & $0.008(2)$ & $0.025(2)$ & $0.008(2)$ \\
\hline$C(5)$ & $4 e$ & $0.6825(3)$ & $0.2574(5)$ & $0.6169(3)$ & $0.052(3)$ & $0.059(3)$ & $0.069(3)$ & $0.008(2)$ & $0.022(2)$ & $0.013(2)$ \\
\hline$C(6)$ & $4 e$ & $0.7418(3)$ & $0.1865(6)$ & $0.6771(3)$ & $0.053(3)$ & $0.094(4)$ & $0.069(3)$ & $0.011(3)$ & $0.012(2)$ & $0.006(3)$ \\
\hline$C(7)$ & $4 e$ & $0.7234(3)$ & $0.0618(6)$ & $0.6981(3)$ & $0.075(4)$ & $0.083(4)$ & $0.062(3)$ & $0.028(3)$ & $0.026(3)$ & $0.026(3)$ \\
\hline$C(8)$ & $4 e$ & $0.6464(3)$ & $0.0090(6)$ & $0.6607(3)$ & $0.087(4)$ & $0.070(4)$ & $0.072(4)$ & $0.014(3)$ & $0.031(3)$ & $0.034(3)$ \\
\hline$C(9)$ & $4 e$ & $0.5858(3)$ & $0.0801(5)$ & $0.6024(3)$ & $0.062(3)$ & $0.059(3)$ & $0.065(3)$ & $0.005(2)$ & $0.023(2)$ & $0.019(2)$ \\
\hline$C(10)$ & $4 e$ & $0.3066(3)$ & $0.3354(6)$ & $0.4471(3)$ & $0.048(4)$ & $0.104(4)$ & $0.067(3)$ & $-0.003(3)$ & $0.024(2)$ & $0.028(3)$ \\
\hline$C(11)$ & $4 e$ & $0.2371(3)$ & $0.3296(6)$ & $0.3722(3)$ & $0.060(4)$ & $0.100(4)$ & $0.083(4)$ & $-0.002(3)$ & $0.028(3)$ & $0.000(3)$ \\
\hline$C(12)$ & $4 e$ & $0.1502(3)$ & $0.3302(5)$ & $0.3712(3)$ & $0.042(3)$ & $0.075(4)$ & $0.077(3)$ & $0.009(2)$ & $0.023(2)$ & $0.014(3)$ \\
\hline$C(13)$ & $4 e$ & $0.0871(3)$ & $0.2736(7)$ & $0.2984(4)$ & $0.073(4)$ & $0.101(5)$ & $0.118(5)$ & $-0.008(3)$ & $0.037(3)$ & $-0.039(4)$ \\
\hline$C(14)$ & $4 e$ & $-0.0001(3)$ & $0.2691(7)$ & $0.2961(4)$ & $0.058(4)$ & $0.123(6)$ & $0.125(5)$ & $-0.024(4)$ & $0.031(3)$ & $-0.039(4)$ \\
\hline$C(15)$ & $4 e$ & $-0.0256(3)$ & $0.4035(8)$ & $0.3101(4)$ & $0.053(4)$ & $0.135(6)$ & $0.118(5)$ & $0.012(4)$ & $0.006(3)$ & $-0.031(4)$ \\
\hline$C(16)$ & $4 e$ & $0.0348(3)$ & $0.4665(8)$ & $0.3822(4)$ & $0.047(4)$ & $0.170(7)$ & $0.148(6)$ & $0.008(4)$ & $0.025(4)$ & $-0.072(5)$ \\
\hline$C(17)$ & $4 e$ & $0.1217(3)$ & $0.4690(6)$ & $0.3843(4)$ & $0.068(4)$ & $0.098(5)$ & $0.098(4)$ & $-0.007(3)$ & $0.017(3)$ & $-0.025(3)$ \\
\hline$C(18)$ & $4 e$ & $0.6024(3)$ & $0.6090(4)$ & $0.3342(2)$ & $0.061(3)$ & $0.041(3)$ & $0.046(3)$ & $0.000(2)$ & $0.028(2)$ & $-0.003(2)$ \\
\hline$C(19)$ & $4 e$ & $0.5337(3)$ & $0.6699(5)$ & $0.2780(3)$ & $0.057(4)$ & $0.061(3)$ & $0.068(3)$ & $-0.004(2)$ & $0.026(2)$ & $0.020(2)$ \\
\hline$C(20)$ & $4 e$ & $0.4522(3)$ & $0.6345(5)$ & $0.2598(3)$ & $0.065(4)$ & $0.062(3)$ & $0.059(3)$ & $0.006(2)$ & $0.020(2)$ & $0.016(2)$ \\
\hline$C(21)$ & $4 e$ & $0.6884(3)$ & $0.6452(4)$ & $0.3460(2)$ & $0.050(3)$ & $0.047(3)$ & $0.043(2)$ & $-0.005(2)$ & $0.026(2)$ & $-0.006(2)$ \\
\hline$C(22)$ & $4 e$ & $0.7069(3)$ & $0.7586(4)$ & $0.3117(3)$ & $0.068(4)$ & $0.046(3)$ & $0.062(3)$ & $-0.003(2)$ & $0.036(2)$ & $-0.006(2)$ \\
\hline$C(23)$ & $4 e$ & $0.7882(3)$ & $0.7868(5)$ & $0.3256(3)$ & $0.076(4)$ & $0.060(3)$ & $0.082(4)$ & $-0.024(3)$ & $0.050(3)$ & $-0.016(3)$ \\
\hline$C(24)$ & $4 e$ & $0.8514(3)$ & $0.7055(6)$ & $0.3718(3)$ & $0.061(4)$ & $0.099(5)$ & $0.077(4)$ & $-0.017(3)$ & $0.038(3)$ & $-0.016(3)$ \\
\hline$C(25)$ & $4 e$ & $0.8339(3)$ & $0.5924(6)$ & $0.4047(3)$ & $0.052(4)$ & $0.098(4)$ & $0.070(3)$ & $0.004(3)$ & $0.030(2)$ & $0.003(3)$ \\
\hline$C(26)$ & $4 e$ & $0.7537(3)$ & $0.5639(5)$ & $0.3924(2)$ & $0.062(4)$ & $0.070(3)$ & $0.054(3)$ & $0.002(2)$ & $0.031(2)$ & $0.003(2)$ \\
\hline$C(27)$ & $4 e$ & $0.3811(3)$ & $0.7011(6)$ & $0.1940(3)$ & $0.078(4)$ & $0.074(4)$ & $0.078(4)$ & $0.001(3)$ & $0.018(3)$ & $0.031(3)$ \\
\hline$C(28)$ & $4 e$ & $0.3442(3)$ & $0.6057(6)$ & $0.1298(3)$ & $0.101(4)$ & $0.069(4)$ & $0.081(4)$ & $0.011(3)$ & $0.027(3)$ & $0.018(3)$ \\
\hline$C(29)$ & $4 e$ & $0.2603(3)$ & $0.6492(5)$ & $0.0658(3)$ & $0.077(4)$ & $0.069(4)$ & $0.070(3)$ & $0.007(3)$ & $0.017(3)$ & $0.019(3)$ \\
\hline$C(30)$ & $4 e$ & $0.2355(4)$ & $0.5473(6)$ & $0.0027(3)$ & $0.084(4)$ & $0.085(4)$ & $0.090(4)$ & $0.002(3)$ & $0.021(3)$ & $0.002(3)$ \\
\hline$C(31)$ & $4 e$ & $0.1534(4)$ & $0.5798(7)$ & $-0.0619(3)$ & $0.089(5)$ & $0.110(5)$ & $0.081(4)$ & $0.008(4)$ & $0.011(3)$ & $-0.009(3)$ \\
\hline$C(32)$ & $4 e$ & $0.0839(4)$ & $0.5922(8)$ & $-0.0369(4)$ & $0.076(5)$ & $0.130(6)$ & $0.111(5)$ & $-0.003(4)$ & $0.011(4)$ & $0.003(4)$ \\
\hline$C(33)$ & $4 e$ & $0.1058(4)$ & $0.6999(8)$ & $0.0248(4)$ & $0.093(5)$ & $0.123(6)$ & $0.111(5)$ & $0.023(4)$ & $0.037(4)$ & $0.000(4)$ \\
\hline$C(34)$ & $4 e$ & $0.1911(4)$ & $0.6684(6)$ & $0.0905(3)$ & $0.122(5)$ & $0.086(4)$ & $0.069(4)$ & $0.006(4)$ & $0.033(4)$ & $0.006(3)$ \\
\hline$O(1)$ & $4 e$ & $0.5663(2)$ & $0.3498(3)$ & $0.4736(2)$ & $0.048(2)$ & $0.052(2)$ & $0.052(2)$ & $0.005(1)$ & $0.024(1)$ & $0.014(1)$ \\
\hline$O(2)$ & $4 e$ & $0.3982(2)$ & $0.4060(3)$ & $0.3933(2)$ & $0.039(2)$ & $0.068(2)$ & $0.059(2)$ & $0.001(1)$ & $0.018(1)$ & $0.017(2)$ \\
\hline$O(3)$ & $4 e$ & $0.5970(2)$ & $0.5181(3)$ & $0.3800(2)$ & $0.043(2)$ & $0.053(2)$ & $0.050(2)$ & $0.000(1)$ & $0.022(1)$ & $0.009(1)$ \\
\hline$O(4)$ & $4 e$ & $0.4289(2)$ & $0.5447(3)$ & $0.2947(2)$ & $0.053(2)$ & $0.073(2)$ & $0.058(2)$ & $-0.002(2)$ & $0.017(1)$ & $0.025(2)$ \\
\hline
\end{tabular}

\section{References}

1. Schilde, U.; Mickler, W.; Uhlemann, E.: Crystal structure of bis(1-phenyldecane-1,3-dionato)copper(II), [Cu(C $\left.\left.\mathrm{C}_{16} \mathrm{H}_{21} \mathrm{O}_{2}\right)_{2}\right]$. Z. Kristallogr. 211 (1996) 503-505.

2. Mickler, W.; Uhlemann, E.: Liquid-liquid extraction of copper from ammoniacal solution with cyclohexyl-substituted $\beta$-diketones. Sep. Sci. Technol. 28 (1993) 2643-2650.

3. Sheldrick, G. M.: SHELXL-93. Program for the refinement of crystal structures. University of Göttingen, Germany 1993. 